\title{
Pelizaeus-Merzbacher-like disease is caused not only by a loss of connexin47 function but also by a hemichannel dysfunction
}

\author{
Simone Diekmann ${ }^{1}$, Marco Henneke ${ }^{1}$, Birgitta C Burckhardt ${ }^{2}$ and Jutta Gärtner ${ }^{\star}$ \\ Autosomal recessive mutations in the GJA12/GJC2 gene encoding the gap junction protein connexin47 (C $\times 47)$ cause a form \\ of Pelizaeus-Merzbacher-like disease (PMLD) with hypomyelination, nystagmus, impaired psychomotor development and \\ progressive spasticity. We investigated the functional consequences of four $\mathbf{C} \times 47$ missense mutations (G149S, G236R, T265A, \\ and T398I) and one $C \times 47$ complex mutation (A98G_V99insT) by immunoblot analysis and immunocytochemistry in transfected \\ communication-incompetent HeLa cells and in OLI-neu cells. All studied C $\times 47$ mutants, except G236R, generated stable \\ proteins in transfected HeLa cells and OLI-neu cells. The mutants T265A and A98G_V99insT were retained in the ER, T398I \\ formed gap junctional plaques at the plasma membrane, and G149S showed both, structures at the plasma membrane and \\ ER localization. Two-microelectrode voltage clamp analyses in Xenopus laevis oocytes injected with wild-type and mutant $\mathrm{C} \times 47$ \\ cRNA revealed reduced hemichannel currents for G236R, T265A, and A98G_V99insT. In contrast, T398I revealed hemichannel \\ currents comparable to wild-type. For $C \times 47$ mutant T398I, our results indicate a defect in hemichannel function, whereas \\ C $\times 47$ mutants G149S, G236R, T265A, and A98G_V99insT are predicted to result in a loss of $\mathrm{C} \times 47$ hemichannel function. \\ Thus, PMLD is likely to be caused by two different disease mechanisms: a loss of function and a dysfunction. \\ European Journal of Human Genetics (2010) 18, 985-992; doi:10.1038/ejhg.2010.61; published online 5 May 2010
}

Keywords: leukodystrophy; Pelizaeus-Merzbacher-like disease; GJA12/GJC2 mutations; connexin47; voltage clamp analysis

\section{INTRODUCTION}

Pelizaeus-Merzbacher Disease (PMD; MIM no. 312080), the prototype of hypomyelinating disorders, is due to X-linked recessive rearrangements or mutations of the proteolipid protein (PLP1) gene. ${ }^{1}$ Clinical features of the classical form comprise nystagmus and impaired psychomotor development within the first months of life followed by progressive spasticity and cerebellar signs. Pelizaeus-Merzbacher-like disease (PMLD, MIM no. 608804) shows clinical and neuroradiologic features, such as classic PMD, but is not associated with PLP1 molecular defects. ${ }^{2}$ Recessive mutations in the GJA12 gene, recently renamed GJC2 (http://www.genenames.org/genefamily/gj.php), cause one form of PMLD. ${ }^{3}$ This gene encodes gap junction protein connexin47 $(\mathrm{C} \times 47)$. Until now 23 different GJA12/GJC2 mutations have been reported. ${ }^{3-9}$

Gap junctions (GJs) are specialized channels between apposed cells allowing direct metabolic and electrical communication between most cell types in mammalian tissues by passive diffusion of molecules smaller than $1000 \mathrm{Da}$. This gap junction intercellular communication (GIIC) has an important role in essential cellular processes, such as development, proliferation, differentiation, and cell death. ${ }^{10}$ Gap junctions are highly specialized structures, consisting of two hemichannels of apposing cells. Several thousand GJ channels assemble to gap junctional plaques. The hemichannel (connexon) in the plasma membrane is formed by six connexins. Connexins, highly conserved integral membrane proteins, have four transmembrane domains, two extracellular loops and three cytoplasmic components, one amino- and carboxy-terminal region, and a cytoplasmic loop. Homotypic GJs consist of hemichannels with one type of connexins, whereas heterotypic GJs are formed by hemichannels with different connexins. ${ }^{11}$ So far, 20 connexin genes have been described in mouse and 21 in human genome. More than half of them are expressed in the nervous system. ${ }^{12}$

Astrocytes and oligodendrocytes are coupled by GJs forming part of the 'panglial syncytium.13 Gap junction intercellular communication occurs between oligodendrocytes and astrocytes $(\mathrm{O} / \mathrm{A})$ and adjacent astrocytes (A/A), but there is no evidence for GJs between adjacent oligodendrocytes in mice. ${ }^{13-16}$ Oligodendrocytes express $\mathrm{C} \times 47$, $C \times 32$, and $C \times 29,{ }^{16,17}$ whereas astrocytes express $C \times 26, C \times 30$, and $\mathrm{C} \times 43 .^{18,19}$ The $\mathrm{O} / \mathrm{A}$ coupling is mediated by heterotypic channels between $C \times 47 / C \times 43$ and $C \times 32 / C \times 30$, both appearing to be essential for the proper maintenance of myelin. ${ }^{20}$ So far, $\mathrm{C} \times 47$ mutants have been shown to result in loss of function, suggesting an impaired O/A coupling mediated by $\mathrm{C} \times 47 / \mathrm{C} \times 43 .{ }^{21}$ Here, we provide further evidence that loss of $\mathrm{C} \times 47$ function causes PMLD. However, our results further suggest a second disease mechanism, namely a $\mathrm{C} \times 47$ hemichannel dysfunction.

\section{MATERIALS AND METHODS}

Wild-type and mutant expression constructs

The full length $C \times 47$ (1-1320 bp, 1-439 aa, RefSeqID NM_020435.2) was PCR amplified from wild-type cDNA and cloned into the mammalian expression

\footnotetext{
${ }^{1}$ Department of Pediatrics and Pediatric Neurology, Georg August University, Göttingen, Germany; ${ }^{2}$ Department of Physiology and Pathophysiology, Georg August University, Göttingen, Germany

${ }^{*}$ Correspondence: Professor J Gärtner, Department of Pediatrics and Pediatric Neurology, Georg August University, Robert-Koch-Strasse 40, 37075 Göttingen, Germany. Tel: +49 551 398035; Fax: +49 551 396252; E-mail: gaertnj@med.uni-goettingen.de

Received 29 June 2009; revised 1 April 2010; accepted 1 April 2010; published online 5 May 2010
} 
vector pcDNA3.1+ (Invitrogen, Karlsruhe, Germany). Site-directed mutagenesis was performed using the FlipFlop Site-Directed Mutagenesis Kit (Bioline, Luckenwalde, Germany). Wild-type $\mathrm{C} \times 47$ with the alternative start codon (10$1320 \mathrm{bp}, 4-439$ aa) was also cloned into pcDNA3.1+.

\section{Generation of $\mathrm{C} \times 47$ antiserum}

Polyclonal rabbit antiserum against human $\mathrm{C} \times 47$ was generated by conventional methods using glutathione-S-transferase (GST) fusion protein as immunogen (Seramun, Heidesee, Germany). A fragment of the intracellular loop (310$591 \mathrm{bp}, 104-197$ aa) was amplified using wild-type $\mathrm{C} \times 47$ construct as template. The PCR product was subcloned into the pGEX-KG vector that encodes for GST. The GST fusion protein was expressed by BL21 bacteria (Bioline) and purified using glutathione-Sepharose4B (GEHealthcare, München, Germany). Final bleed serum was used for immunoblot analysis at dilution 1:10 000. This antiserum did not work properly in immunoblot analysis of oocyte lysates.

\section{Immunoblot analysis}

HeLa cells were cultured in Dulbecco's modified Eagle's medium containing $10 \%$ fetal bovine serum at $37^{\circ} \mathrm{C}$ and $5 \% \mathrm{CO}_{2}$. Transfection was carried out using the Effectene Kit (Qiagen, Hilden, Germany). Transfected HeLa cells were collected at $48 \mathrm{~h}$ after transfection and lysed in RIPA buffer $(150 \mathrm{~mm} \mathrm{NaCl}$; 50 mm Tris-HCl; 5 mm EDTA; 0.5\% Na-deoxycholate; $1 \%$ NP-40; 0.1\% SDS; protease inhibitor cocktail). Immunoblot analyses were performed using standard techniques. Horseradish peroxidase-conjugated secondary antibodies were used to view bound primary antibodies $(\mathrm{C} \times 47$ antiserum) using Lumi-Light Western Blotting Substrate (Roche, Mannheim, Germany). Chemiluminescence signals were analysed using the Luminescent Image Analyzer, LAS-4000mini (Fujifilm, Düsseldorf, Germany).

\section{Immunocytochemistry}

An immortalized oligodendrocyte precursor cell line, OLI-neu, ${ }^{22}$ was cultured in Sato medium containing $1 \%$ horse serum on poly-(L-lysine)-coated culture dishes at $37^{\circ} \mathrm{C}$ and $5 \% \mathrm{CO}_{2}$. The OLI-neu cells were transfected with
FuGENE-HD (Roche, Germany). HeLa and OLI-neu cells transfected with wild-type or mutant $\mathrm{C} \times 47$ were fixed at $20-48 \mathrm{~h}$ after transfection with $4 \%$ paraformaldehyde/PBS. Immunostaining was done using standard methods with the $\mathrm{C} \times 47$ antiserum (dilution 1:2000) and with anti-Pan-Cadherin (plasma membrane marker, Abcam, Cambridge, UK) and anti-PDI (ER marker, Abcam) antibodies to detect co-localization. Alexa488- and Cy3-conjugated anti-rabbit and anti-mouse secondary antibodies were used (Molecular Probes, Karlsruhe, Germany and Jackson ImmunoResearch, Suffolk, UK; diluted 1:1000 and 1:500, respectively). The cells were embedded in ProLongGold mounting medium with DAPI (Invitrogen). Fluorescence signals were detected using fluorescence microscope Axio-Imager M.1 (Zeiss, Göttingen, Germany).

\section{Oocyte preparation and microinjection}

The stage V and VI oocytes from Xenopus laevis (Nasco, Fort Atkinson, WI, USA) were separated by treatment with collagenase (TypCLS II, Biochrom, Berlin, Germany) and maintained at $16-18^{\circ} \mathrm{C}$ in a daily-replaced control solution (in mM): $110 \mathrm{NaCl}, 3 \mathrm{KCl}, 2 \mathrm{CaCl}_{2}, 5$ HEPES ( $\mathrm{pH} 7.5$ ), supplemented with sodium pyruvate $(0.55 \mathrm{mg} / \mathrm{ml})$ and gentamycin $(50 \mu \mathrm{g} / \mathrm{ml})$. An antisense oligonucleotide complementary to Xenopus $\mathrm{C} \times 38 \mathrm{mRNA}(\mathrm{asC} \times 38)$ was synthesized: 5 '-gctttagtaattcccatcctgccatgtttcc-3' (Metabion, Martinsried, Germany). ${ }^{23}$ To deplete endogenous $\mathrm{C} \times 38$ expression, as $\mathrm{C} \times 38$ was injected $(23 \mathrm{nl}, 1 \mathrm{ng} / \mathrm{nl})$ into oocytes 1 day after the removal from the frog and $48-72 \mathrm{~h}$ before recording. To analyze the function of $\mathrm{C} \times 47$ wild-type and mutant hemichannels in oocytes, cRNA transcription was performed in vitro, using the SP6 mMessage mMachine Kit (Ambion, Foster City, CA, USA). The cRNA ( $5 \mathrm{ng}$ per oocyte) was co-injected with as $\mathrm{C} \times 38$.

\section{Electrophysiological studies}

Resting membrane potentials and non-junctional transmembrane currents in single oocytes were studied by two-electrode voltage clamp (TEVC) technique using a commercial amplifier (OC-725C, Warner, Hambden, CT, USA). The microelectrodes were filled with $3 \mathrm{M} \mathrm{KCl}$ and had resistances of less than $1 \mathrm{M} \Omega$. Oocytes were superfused with external bath solution (control solution without
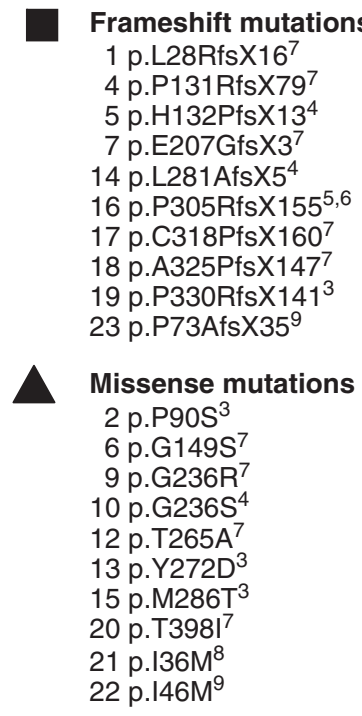

Nonsense mutations
8 p.Y232X
11 p.R240X 11 p. $240 X^{3}$ 3 p.A98G_V99ins $T^{7}$
Missense/Insertion

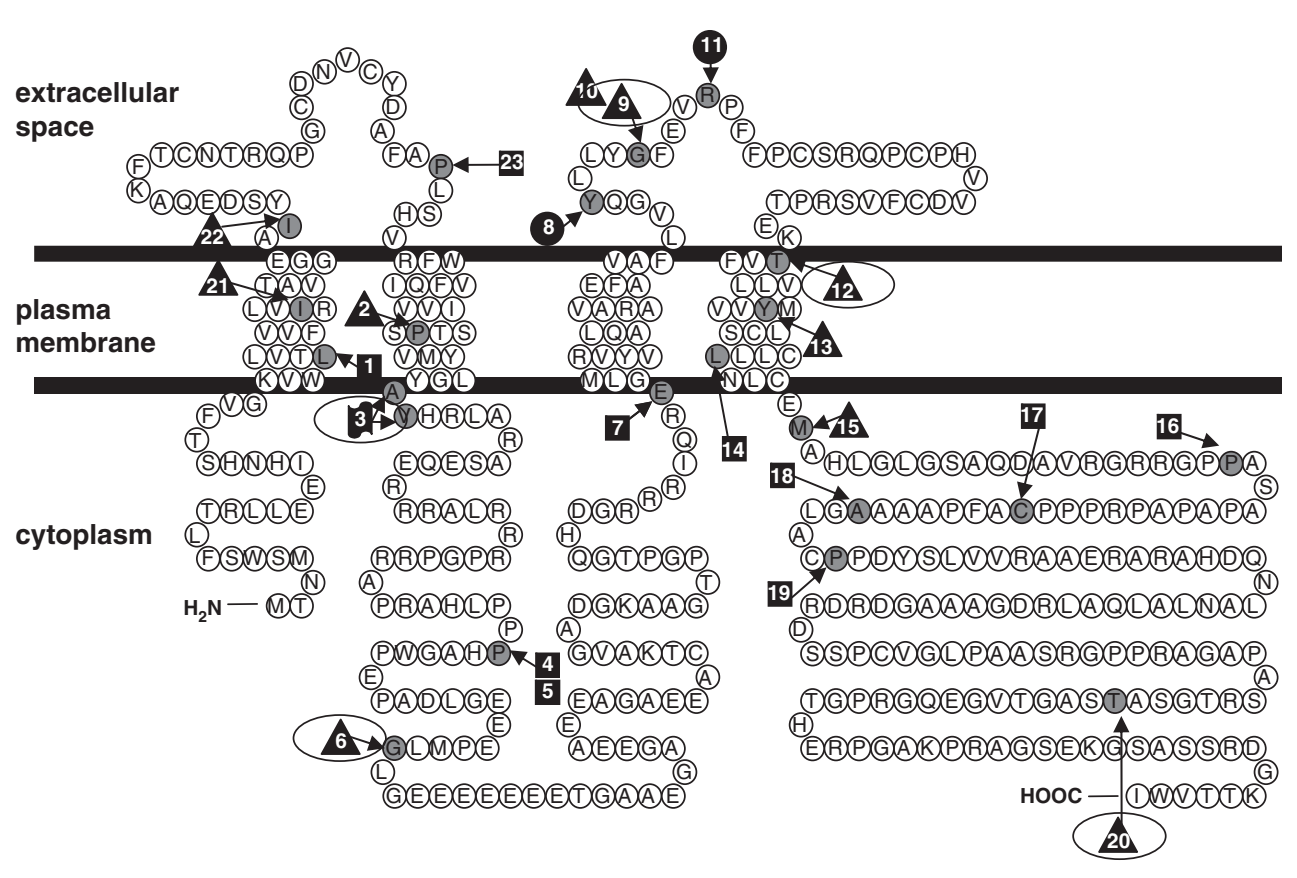

Figure 1 Membrane topology and localization of all known GJA12/GJC2 mutations. Seven mutations are observed on the four membrane-spanning domains, six on the intracellular loop, four on the extracellular loop, and six on the cytoplasmic carboxy-terminus. The five mutations characterized in this study are encircled. Mutations are designated according to current guidelines of international mutation nomenclature. Designation of the mutation p.P305RfsX1556 and of the mutation p. P73Afs $X 35^{9}$ was changed according to current guidelines of mutation nomenclature. 
sodium pyruvate and gentamycin). After stabilization of the membrane potential, single oocytes were clamped to $-40 \mathrm{mV}$. Superfusion was switched after $200 \mathrm{~s}$ to calcium-free bath solution. Non-junctional transmembrane currents were measured after $5 \mathrm{~min}$. A depolarization pulse to $0 \mathrm{mV}$ was applied to the oocyte and transmembrane currents were measured after 100 s. All currents were measured at a saturation of about $100 \%$ (see Supplementary Figure 1). At $-40 \mathrm{mV}$, calcium-activated chloride currents are mostly inactivated. ${ }^{24}$ Channel opening is blocked by external divalent cations (ie, $\mathrm{Ca}^{2+}$ ) and in most cases by hyperpolarizing transmembrane potentials. ${ }^{25}$

The data were corrected by subtracting the average leakage current measured in control oocytes (only injected with as $\mathrm{C} \times 38$ ). Some outliers were excluded using the Nalimov outlier test. An unpaired Student's $t$-test was probed.

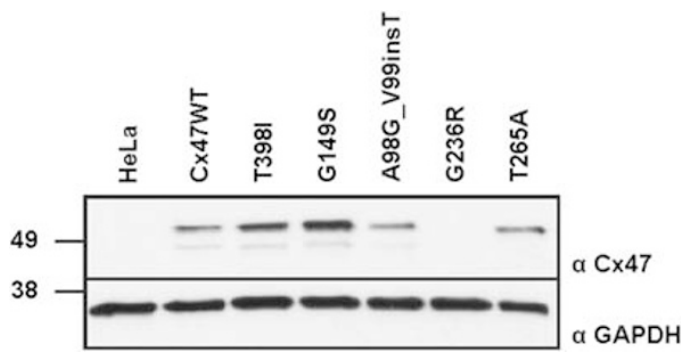

Figure 2 Immunoblot analysis of $\mathrm{C} \times 47$ expression in transfected communication incompetent HeLa cells. Immunoblot analysis was probed with HeLa cells transiently transfected with wild-type $\mathrm{C} \times 47 \quad(\mathrm{C} \times 47 \mathrm{WT})$, mutant C×47 (T398I, G149S, A98G_V99insT, G236R, and T265A) and with untransfected HeLa cells (HeLa). Immunoblots were probed with a rabbit antiserum against human $\mathrm{C} \times 47(1: 10000)$ and a monoclonal GAPDH antibody (Abcam, 1:5000).

\section{RESULTS}

Expression of wild-type and mutant $\mathrm{C} \times 47$ in HeLa cells

We analyzed the functional consequences of four missense mutations G149S, G236R, T265A, and T398I and one complex mutation A98G_V99insT with an amino-acid substitution followed by an amino-acid insertion, as previously described. ${ }^{7}$ The localization within the different protein domains is illustrated in Figure 1 and is one criterion to study these five distinct missense mutations. To determine whether selected mutations affect protein expression, immunoblot analysis of transfected HeLa cells was performed (Figure 2). The wildtype and all mutants except G236R were detected by the $\mathrm{C} \times 47$ antiserum. There was no signal in untransfected HeLa cells, indicating the specificity of the $\mathrm{C} \times 47$ antiserum.

\section{Intracellular localization of wild-type and mutant $\mathrm{C} \times 47$ in HeLa and OLI-neu cells}

A second alternative $\mathrm{C} \times 47$ start codon, nine nucleotides downstream from the first ATG, was described. ${ }^{21}$ We analyzed the expression and localization of the constructs with the first and the second start codon by immunostaining transfected HeLa and OLI-neu cells. $\mathrm{C} \times 47$ proteins were immunolabeled with the $\mathrm{C} \times 47$ antiserum and visualized using epifluorescence microscopy (Figure 3 ). There was no difference in subcellular localization: both proteins were found in punctate structures localized to the plasma membrane. Therefore, all constructs analyzed in this study were cloned starting from the first ATG. Accordingly, our description of nucleotide and amino-acid positions, including the designation of mutations refers to the first upstream ATG.
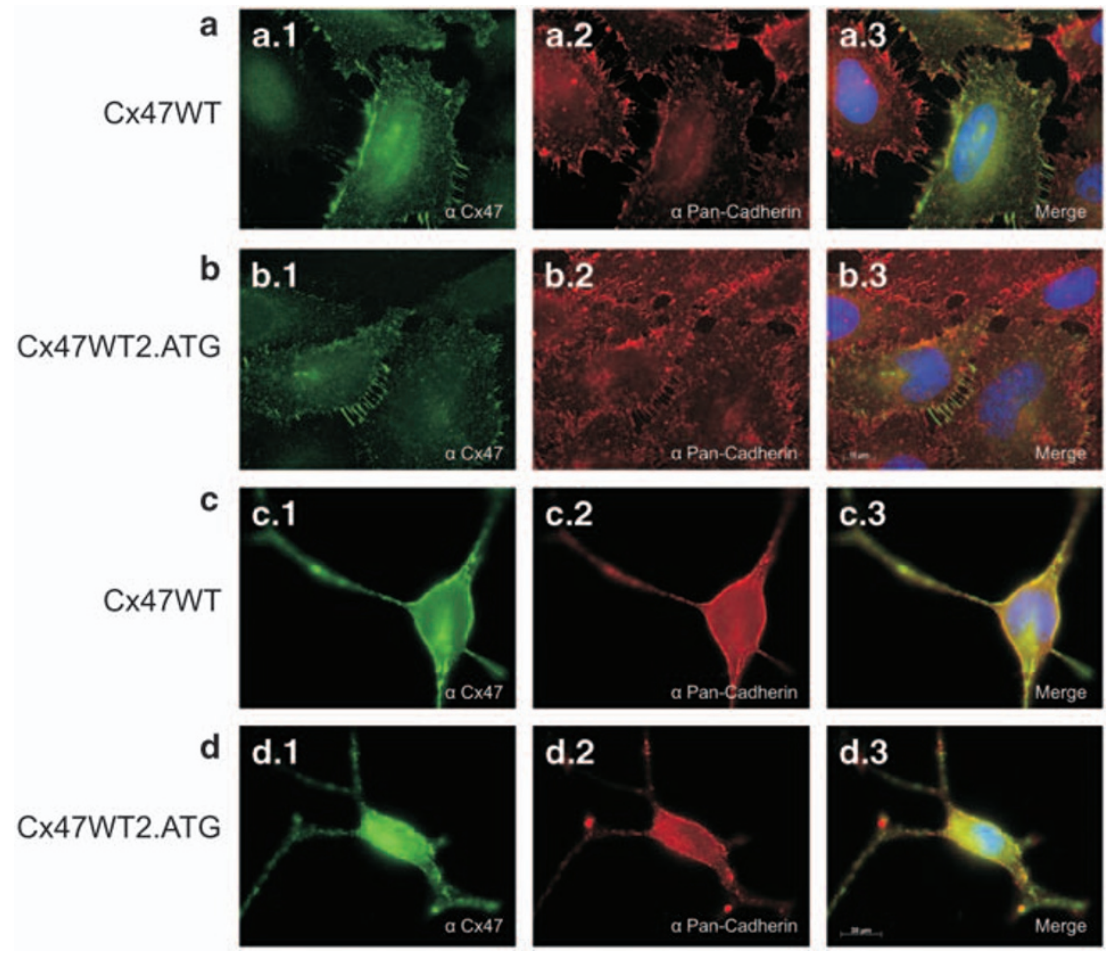

Figure 3 Subcellular localization of wild-type $C \times 47$. Double-staining immunofluorescence microscopy studies in communication incompetent HeLa cells (a and $\mathbf{b}$ ) and OLI-neu cells (c and $\mathbf{d}$ ) transiently transfected with wild-type $\mathrm{C} \times 47$ first ATG $(\mathrm{C} \times 47 \mathrm{WT}$; $\mathbf{a}$ and $\mathbf{c}$ ) and alternative second ATG (C $\times 47$ WT2.ATG; b and d). Transfected cells were immunolabeled with a rabbit antiserum against human $\mathrm{C} \times 47$ (green) and a mouse monoclonal antibody against the plasma membrane protein Pan-Cadherin (red). Gap junctional plaques were seen at the plasma membrane for cells expressing wild-type $\mathrm{C} \times 47$ from the first ATG and for cells expressing wild-type $\mathrm{C} \times 47$ from the alternative second ATG. 


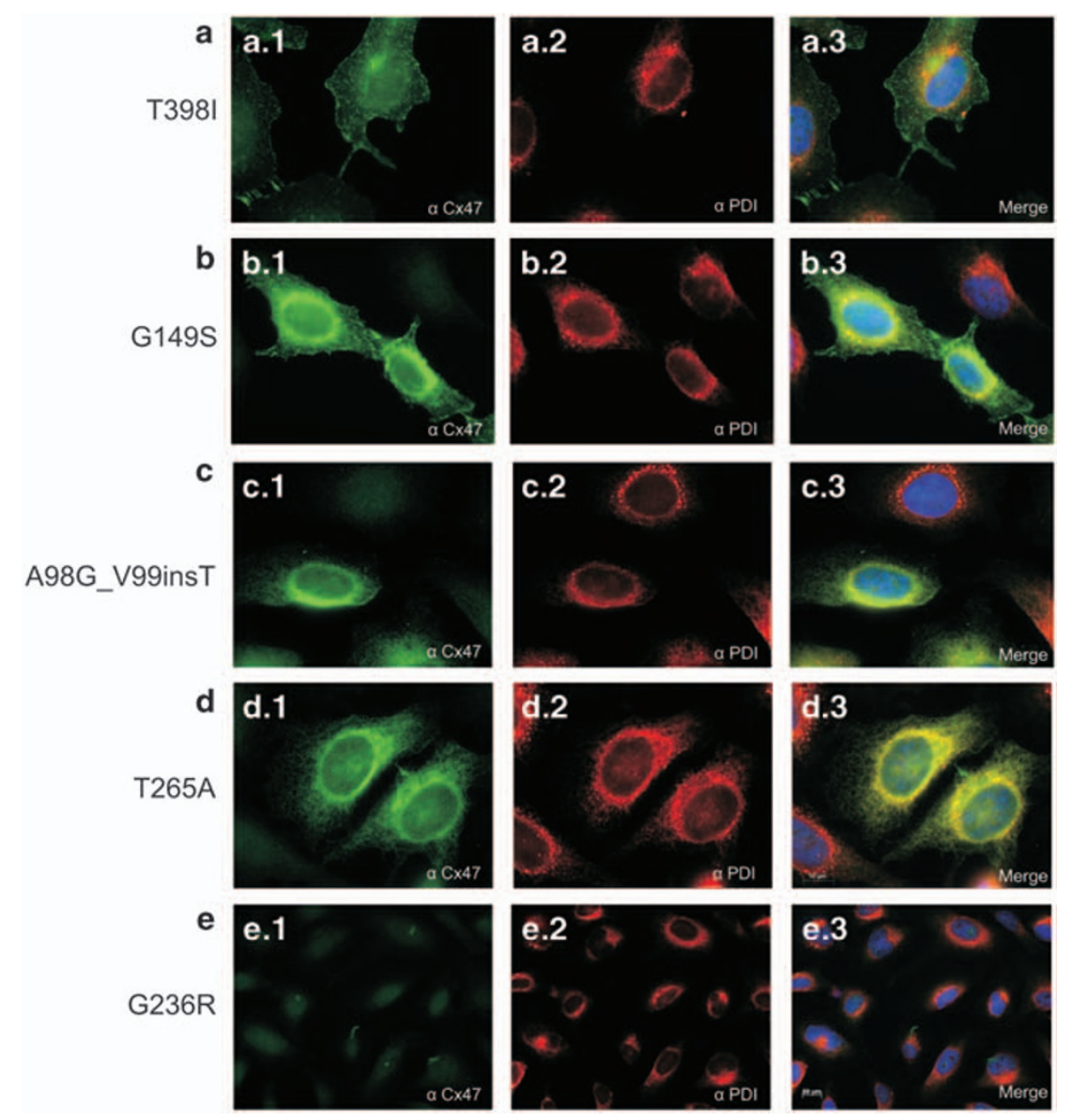

Figure 4 Subcellular localization of mutant $\mathrm{C} \times 47$ in HeLa cells. Communication incompetent HeLa cells transiently transfected with mutant $\mathrm{C} \times 47$ ( $\mathrm{T398I}$, G149S, A98G_V99insT, T265A, and G236R) were immunolabeled with a rabbit antiserum against human $\mathrm{C} \times 47$ (green) and a mouse monoclonal antibody against the ER protein disulfide isomerase ( $\alpha$ PDI, red). Gap junctional plaques were seen at the plasma membrane for cells expressing mutant T398I and G149S (a and b). There was also partial co-localization with the ER marker for both mutants, but the ER co-localization was even higher for the mutant G149S. Nearly perfect ER co-localization was seen for the mutants A98G_V99insT and T265A (c and d). As expected, there was no signal for mutant G236R (e).

To examine whether the $\mathrm{C} \times 47$ mutants were properly targeted to the plasma membrane, HeLa and OLI-neu cells, transfected with one of the mutant constructs, were immunolabeled with the $\mathrm{C} \times 47$ antiserum (Figures 4 and 5). Double-staining immunofluorescence studies revealed differences in the subcellular localization of $\mathrm{C} \times 47$ mutants compared with wild-type protein. As expected, wild-type $\mathrm{C} \times 47$ was found in punctate structures co-localizing to the plasma membrane. These structures are assumed to represent gap junctional plaques. The mutant, T398I, was labeled as punctate structures predominantly at the plasma membrane (Figures $4 \mathrm{a}$ and $5 \mathrm{a}$ ). However, there was also a faint co-localization to the ER. HeLa and OLI-neu cells expressing G149S showed punctate structures at the plasma membrane and in the ER (Figures $4 \mathrm{~b}$ and $5 \mathrm{~b}$ ). The mutants A98G_V99insT and T265A were only localized to the ER (Figures $4 c, d, 5 c$ and d). As expected, there was no fluorescence signal in G236R-transfected (Figures $4 \mathrm{e}$ and 5e) and in untransfected HeLa and OLI-neu cells (data not shown).

\section{Functional characterization of wild-type and mutant $\mathrm{C} \times 47$ in} Xenopus laevis oocytes

Resting membrane potentials and non-junctional transmembrane currents in single oocytes were studied by two-electrode voltage clamp (TEVC) technique. Average resting membrane potentials upon superfusion with calcium-containing solution for oocytes injected with as $\mathrm{C} \times 38$ and wild-type $\mathrm{C} \times 47$ cRNA (WT) or mutant C $\times 47$ cRNA (G149S, A98G_V99insT, G236R, and T265A) ranged from $-35.4 \pm 0.8$ to $-39.2 \pm 0.9 \mathrm{mV}$ (Figure 6a). There was no significant change of resting membrane potential compared with wild type; only for those 84 oocytes injected with T398I, a significant decreased potential was observed $(-30.4 \pm 0.9 \mathrm{mV}, P=0.0001)$. Resting membrane potential from 79 control oocytes showed a small significant hyperpolarization $(-41.9 \pm 0.9 \mathrm{mV}$, compared with wild-type $-39.2 \pm 0.9 \mathrm{mV}, P=0.025)$. The injected oocytes were clamped to $-40 \mathrm{mV}$ and superfused with calcium-free solution. They showed slowly activated inward currents that were reversibly inactivated when calcium-free solution was replaced. The amplitude of wild-type calcium-sensitive hemichannel currents after subtracting the average leakage current measured in control oocytes was $-554 \pm 49 \mathrm{nA}$ $\left(\Delta I_{\mathrm{m}}\right.$, Figure 6b). In T398I-injected oocytes, $\Delta I_{\mathrm{m}}$ represented $-693 \pm 58 \mathrm{nA}$. There is no significant difference between corrected wild-type and T398I hemichannel currents $(P=0.0826)$. Significantly reduced transmembrane currents were observed for G149S, A98G_V99insT, G236R, and T265A $(P \leq 0.0012$, compared with wild-type). Application of depolarization step to $0 \mathrm{mV}$ elicited an 


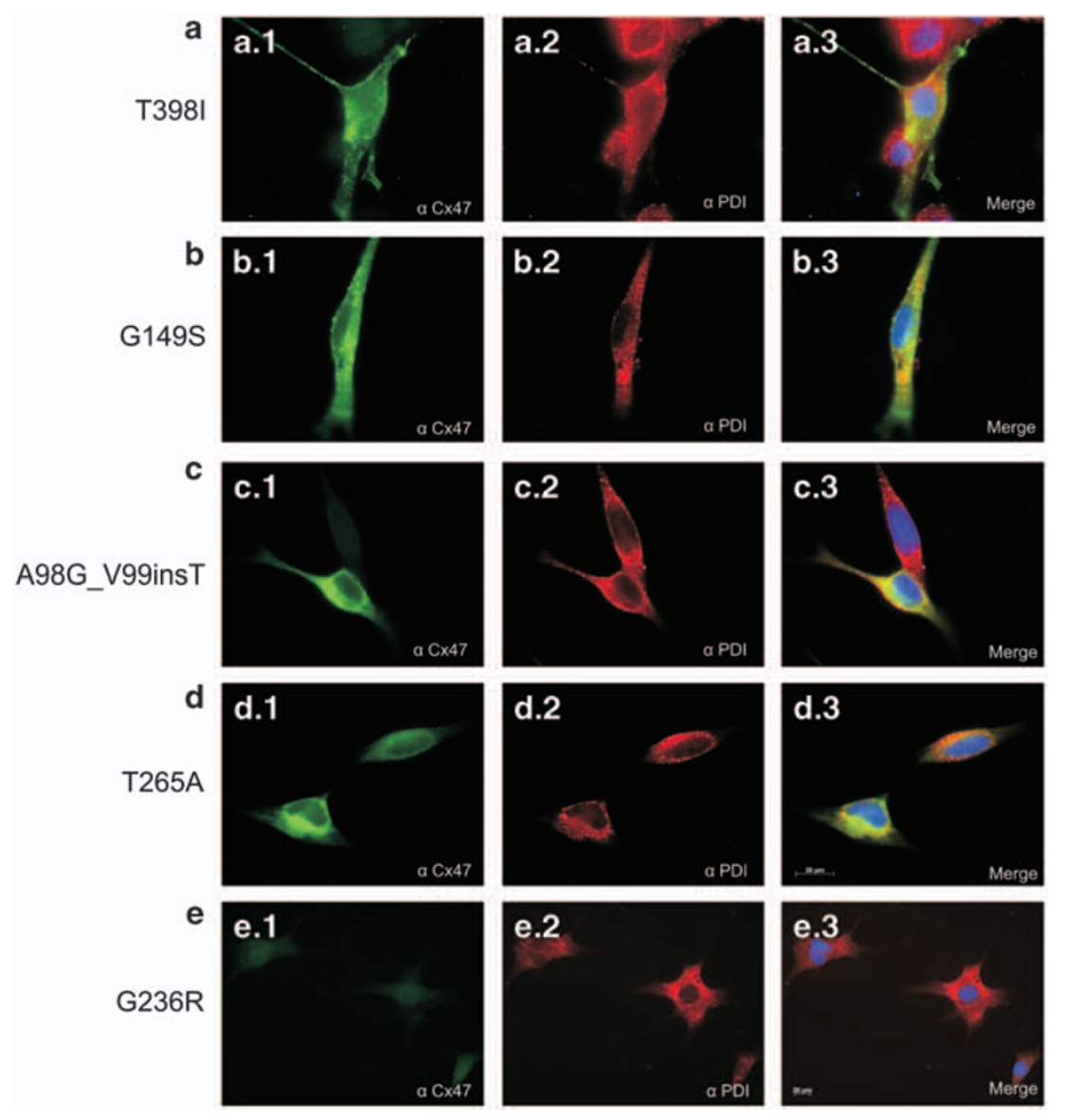

Figure 5 Subcellular localization of mutant $\mathrm{C} \times 47$ in OLI-neu cells. OLI-neu cells transiently transfected with mutant C $\times 47$ (T398I, G149S, A98G_V99insT, T265A, and G236R) were immunolabeled with a rabbit antiserum against human $\mathrm{C} \times 47$ (green) and a mouse monoclonal antibody against the ER protein disulfide isomerase ( $\alpha$ PDI, red). Similar to in HeLa cells gap junctional plaques were observed at the plasma membrane for OLI-neu cells expressing mutant T398I and G149S (a and $\mathbf{b}$ ). There was also partial co-localization with the ER marker for both mutants, but the ER co-localization was even higher in the mutant G149S. Nearly perfect ER co-localization was seen for the mutants A98G_V99insT and T265A (c and d). As expected, no signal for mutant G236R could be detected (e).

instantaneous increment of outward calcium-sensitive currents in oocytes, injected with wild-type $\mathrm{C} \times 47 \mathrm{cRNA}\left(\Delta I_{\mathrm{m}}=406 \pm 36 \mathrm{nA}\right.$, Figure 6c). No depolarizing activation was observed for T398I hemichannel currents average $\left(\Delta I_{\mathrm{m}}=162 \pm 18 \mathrm{nA}\right)$. Transmembrane currents observed for G149S, A98G_V99insT, G236R, and T265A were significantly reduced (below $75 \mathrm{nA} ; P=0.0001$ ). The transmembrane currents varied considerably in magnitude, indicating that conductance of oocytes changed according to the cRNA injected.

\section{DISCUSSION}

A second alternative start codon for $\mathrm{C} \times 47$, nine nucleotides downstream from the first ATG, was described. ${ }^{21}$ So far, there is no experimental evidence regarding which ATG of $\mathrm{C} \times 47$ is the translation initiation codon. Therefore, we analyzed the expression and subcellular localization of both using constructs with the first and the second start codon. Interestingly, we saw no difference in subcellular localization. Both proteins localized to the plasma membrane in HeLa cells. Whether one or both start codons are translated and whether the downstream ATG is more favourable is still unknown. ${ }^{26}$ Furthermore, it needs to be investigated whether a tissue-specific translation exists. Reference sequence NM_020435.2 (NCBI database) declares the upstream ATG to be the putative start codon. All constructs analyzed in this study were cloned starting from the first ATG.
Besides G149S, all studied missense mutations affect highly conserved amino acids, indicating evolutionarily constrained key residues for protein function. ${ }^{7}$ All $\mathrm{C} \times 47$ mutants, except G236R, were able to generate stable proteins in transfected HeLa cells. The G236R mutation leads to an amino-acid substitution of neutral glycine against alkaline arginine in the second highly conserved extracellular domain. There are six highly conserved cysteines in both extracellular domains thought to be essential for connexin stabilization, connexin formation, and connexon docking. ${ }^{27}$ The G236R substitution is located close to C245. Presumably, this mutation inhibits the formation of an important disulfide bond and leads to instability of $\mathrm{C} \times 47$, resulting in early degradation during translation. The degradation results in lack of this protein in the plasma membrane and may inhibit $\mathrm{C} \times 47 / \mathrm{C} \times 43$ heterotypic GJIC between oligodendrocytes and astrocytes. ${ }^{20}$ For PMD, it was shown that mutations mapping into the extracellular loop region of PLP/DM20 lead to the failure of oligodendrocytes to form correct intramolecular disulfide bonds and result in activation of the unfolded protein response (UPR) ${ }^{28}$ Interestingly, point mutations in the second extracellular domain of $\mathrm{C} \times 43$ are known to cause Oculodentodigital Dysplasia (ODDD) preventing $\mathrm{C} \times 43$ localization to the plasma membrane and consecutive loss of function. ${ }^{29}$

The mutation, T398I, located in the C-terminus, does not alter the localization of $\mathrm{C} \times 47$ to the plasma membrane and apparently does not inhibit hemichannel function. The C-terminus contains multiple 
a

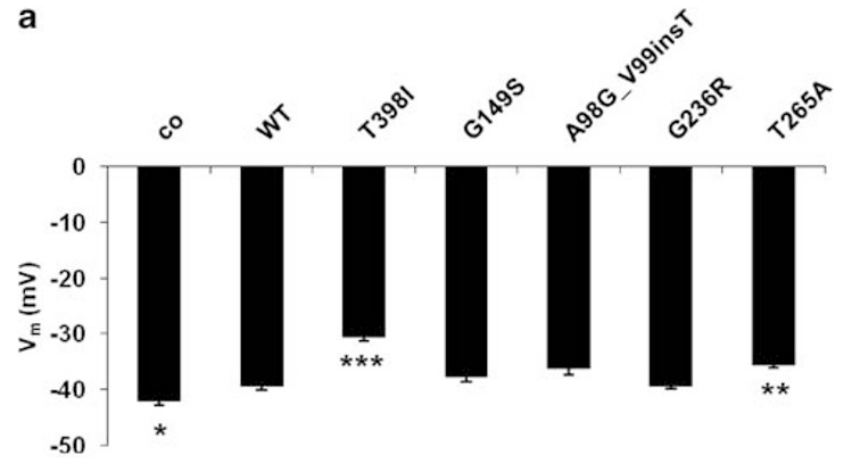

b
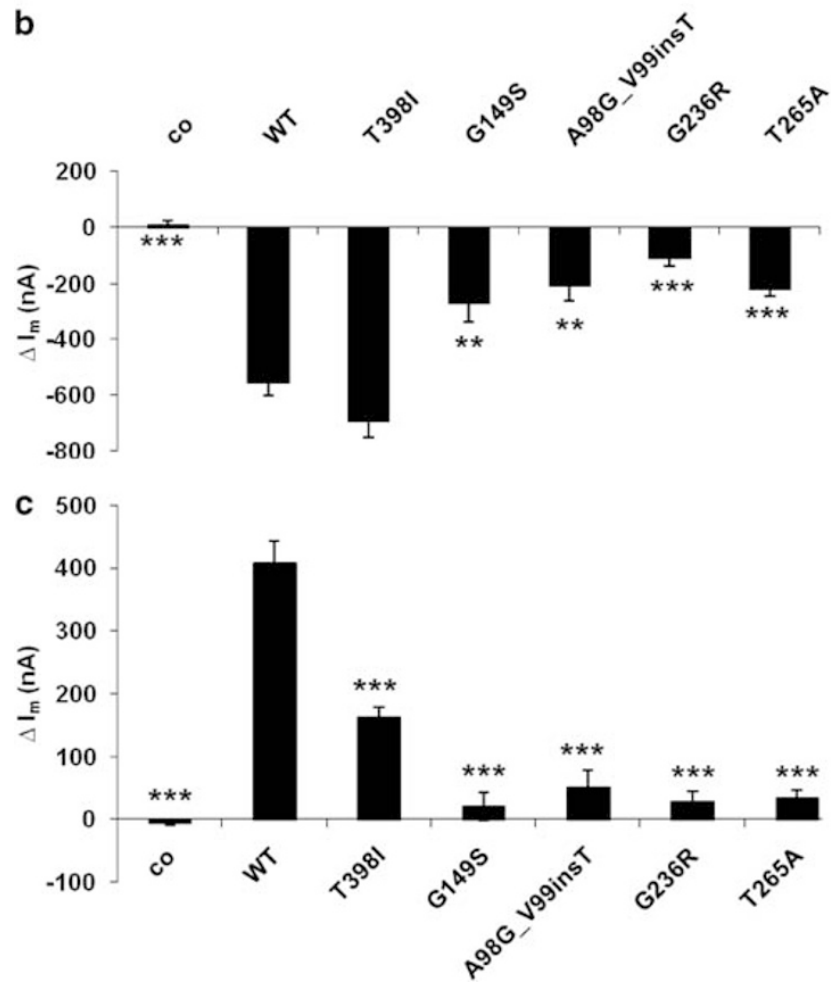

Figure 6 Electrophysiological studies of oocytes injected with wild-type and mutant $\mathrm{C} \times 47$ CRNA. Resting membrane potentials and non-junctional transmembrane currents were measured by two-electrode voltage-clamp techniques. Resting membrane potentials $\left(V_{\mathrm{m}}\right)$ were recorded in control oocytes (co, injected with as $\times 38$ only, $n=79$ ), wild type (WT, $n=84$ ) and mutant (T398I, $n=84 ;$ G149S, $n=37 ;$ A98G_V99insT, $n=34$; G236R, $n=40$; and T265A, $n=53$ ) $\mathrm{C} \times 47$-expressing oocytes (in addition, injected with as $\mathrm{C} \times 38$ ) during perfusion with calcium-containing solution (a). Membrane currents were monitored in control oocytes, wild-type, and mutant $\mathrm{C} \times 47$-expressing oocytes (additionally injected with $\mathrm{asC} \times 38$ ) clamped at $-40 \mathrm{mV}$ (b) or at $0 \mathrm{mV}$ (c) during perfusion with calcium-free solution. All experimental data are averages of different numbers of oocytes ( $n=34-84)$ from at least three different donors. The data were corrected for leakage by subtracting the average leakage current measured in control oocytes $(\Delta / \mathrm{m})$. Statistical analysis was done by an unpaired Student's $t$-test compared with wild-type. Errors are given as SEM. The $P$-values ( $\left.{ }^{*} P \leq 0.026 ;{ }^{*} P \leq 0.0032 ;{ }^{* *} P=0.0001\right)$ were adjusted according to Bonferroni.

phosphorylatable serine, threonine, and tyrosine residues that are considered an intrinsic part of the voltage and low $\mathrm{pH}$ gate for various GJ channels. ${ }^{30}$ The $\mathrm{C}$-terminal mutations in the $C \times 43$ gene were identified in patients with heart malformation and defects in laterality. ${ }^{31}$ In these cases, one or more C-terminal phosphorylatable serine or threonine residues were substituted. Mutant transfectants were demonstrated to have normal levels of GJIC but abnormalities in its regulation. Furthermore, mutations in the C-terminus of $C \times 32$ are thought to affect functional regulation of GJs. ${ }^{32,33}$ Using TEVC, we observed the depolarization of resting membrane potential in oocytes injected with T398I. This observation leads to the hypothesis that T398I hemichannel opening occurs incidentally. Consequently, this mutant may affect oligodendrocytes causing leakage across the plasma membrane through opening hemichannels. Presence of open hemichannels was shown for other connexins and postulated as a possible mechanism of cell injury and cell death in other tissues. ${ }^{34,35}$ Therefore, a regulation defect of $\mathrm{C} \times 47$ hemichannel function in mutant T398I is likely.

Mutation G149S lies within the less conserved intracellular protein domain. It co-localized to the ER and partially also to the plasma membrane. Hemichannel currents were nearly absent. The weak transmembrane currents could be caused by the formation of nonfunctional hemichannels, by aggregation of connexins in the ER preventing the assembly of hemichannels at the plasma membrane, or by unspecific currents higher than those for control oocytes. Faint transmembrane currents were also observed for other mutants localized to the ER (T265A and A98G_V99insT) and for G236R that did not express any protein product. Thus, these currents represent rather unspecific currents.

Both mutations, T265A and A98G_V99insT, located in the transmembrane domains, showed significantly reduced hemichannel currents and were not able to form homomeric hemichannels. Both were retained in the ER. The mutations, P90S, Y272D, and M286T, also located close to or directly at the transmembrane domains, showed aggregation of mutated connexins in the ER and loss of $\mathrm{C} \times 47$ function. ${ }^{21}$ For the transmembrane domains three and four of $\mathrm{C} \times 32$, an important role in protein trafficking has been proposed. ${ }^{36}$ Therefore, mutations in the transmembrane domains of $\mathrm{C} \times 47$ might basically inhibit protein transport.

Two alternative pathomechanisms are considered to be responsible for GJA12/GJC2-associated PMLD. Similar to several previously described alterations our mutants: G149S, G236R, T265A, and A98G_V99insT cause PMLD most likely by loss of $\mathrm{C} \times 47$ function. In contrast, T398I does not lead to loss of function but might result in a hemichannel dysfunction, indicating a second and new disease mechanism in GJA12/GJC2-associated PMLD.

Clinical features of patients carrying the characterized mutations previously described by our group are listed in Table 1 . Mutations G149S, T265A, and A98G_V99insT were found at heterozygous state in four families; thus a genotype-phenotype correlation cannot be done for these alterations as the second allele may modulate the expression of the first one. Mutations T265A and G236R were identified at homozygous state in two families. Both alterations result indeed in a loss of $\mathrm{C} \times 47$ function, but only mutant G236R was not detected as stable protein in our experiments. The residual protein expressed for mutant T265A likely results in the milder phenotype of subject G330. However, more homozygous patients and the functional consequences of their mutations must be analyzed to definitely assess a genotype-phenotype correlation in this disorder.

Mutants P90S, Y272D, and M286T do not efficiently form functional heterotypic $\mathrm{C} \times 47 / \mathrm{C} \times 43 \mathrm{GJs}$, suggesting that disruption of these channels affects human myelination and cause GJA12/GJC2associated PMLD. ${ }^{20,37}$ Mice lacking $\mathrm{C} \times 47$ were viable and fertile and showed no obvious morphological or behavioral abnormalities, ${ }^{17}$ whereas animals lacking $\mathrm{C} \times 47$ and $\mathrm{C} \times 32$ developed a profound CNS demyelination. ${ }^{16}$ Potentially, $\mathrm{C} \times 30 / \mathrm{C} \times 32$ could compensate 
the lack of $C \times 43 / C \times 47$ GJs in mice. ${ }^{20}$ However, there is no evidence for such a compensatory mechanism in humans. Furthermore, $\mathrm{C} \times 47 / \mathrm{C} \times 43$ and $\mathrm{C} \times 32 / \mathrm{C} \times 30$ channels were described to have different roles in $\mathrm{O} / \mathrm{A}$ coupling in primates. ${ }^{37}$ Thus, PMLD is likely caused by loss of function for mutants expressing no proteins and for mutants retained in the ER. However, mutants assembling in the ER, as shown for T265A and A98G_V99insT in our study and as previously shown for P90S, Y272D, and M286T, ${ }^{21}$ could also induce a pathological activation of UPR, resulting in changes of cell physiology (ie apoptosis, abnormal differentiation, and altered proliferation). Although no experimental evidence for this putative pathomechanism was found in a cell model system analyzing the PERK-UPR pathway, the authors Orthmann-Murphy et al. did not rule out that $\mathrm{C} \times 47$ activates a different UPR-related pathway. ${ }^{21}$ For PLP1 mutants causing PMD and for mutants of peripheral myelin protein (PMP22) causing Charcot-Marie-Tooth type 1A (CMT1A) chronic, pathological activation of UPR by accumulation of misfolded and undegraded proteins in the ER has been postulated. ${ }^{38-41}$ The activation of UPR by certain PLP1 mutants may result in oligodendrocyte cell death affecting the myelination process. ${ }^{42}$ Toxic accumulation of $\mathrm{C} \times 32$ in the ER has also been suggested as a pathomechanism for some X-linked CharcotMarie-Tooth (CMTX) $\mathrm{C} \times 32$ mutants. ${ }^{36}$ These authors postulated that inherited diseases of myelin share common pathophysiology in which mutant protein retained in intracellular compartments has deleterious effects on the protein folding, degradation, or trafficking machineries. Potentially, there is co-action of both, loss of ability to form O/A heterotypic GJs and toxic ER accumulation. Moreover, it is possible that oligodendrocyte and astrocyte connexins have roles besides forming GJs. ${ }^{37}$

The O/O coupling in humans has not been examined, so it might be possible that $\mathrm{C} \times 47$ GJs exist between adjacent oligodendrocytes unlike shown in rodents. ${ }^{37}$ In addition to $\mathrm{C} \times 47 / \mathrm{C} \times 43$ dysfunction, abnormal homotypic $\mathrm{C} \times 47$ GJs between oligodendrocytes and myelin sheets could be responsible for the hypomyelination observed in PMLD patients.

Recently, complicated hereditary spastic paraplegia has been described as a new milder phenotype for $\mathrm{C} \times 47$ mutation I33M. ${ }^{8}$ The mutant formed homotypic gap junctional plaques in HeLa cells but formed altered heterotypic channels with $\mathrm{C} \times 43$ in a cell model system. The authors predict that $\mathrm{I} 33 \mathrm{M}$ causes loss of function mutation and disrupts $\mathrm{GJIC}$ via $\mathrm{C} \times 47 / \mathrm{C} \times 43$ channels. If $\mathrm{I} 33 \mathrm{M}$ has the same functional consequences as other mutations, another mechanism must account for the milder phenotype described. ${ }^{8}$

Finally, we could demonstrate a new alternative PMLD pathomechanism, namely a $\mathrm{C} \times 47$ hemichannel dysfunction caused by a mutation localized in the C-terminus. Further studies on C-terminal mutations will contribute to a better understanding of pathomechanisms in PMLD.

\section{CONFLICT OF INTEREST}

The authors declare no conflict of interest.

\section{ACKNOWLEDGEMENTS}

We thank J Kaiser and I Markmann for technical assistance and H Werner and P De Monasterio for providing OLI-neu cells. This study was supported by the Deutsche Forschungsgemeinschaft grant number GA354/6-1 (to JG and MH).

1 Saugier-Veber $\mathrm{P}$, Munnich A, Bonneau $\mathrm{D}$ et al: X-linked spastic paraplegia and Pelizaeus-Merzbacher disease are allelic disorders at the proteolipid protein locus. Nat Genet 1994; 6: 257-262. 
2 Schiffmann R, Boespflug-Tanguy O: An update on the leukodystrophies. Curr Opin Neurol 2001; 14: 789-794.

3 Uhlenberg B, Schuelke M, Rüschendorf $\mathrm{F}$ et al: Mutations in the gene encoding gap junction protein alpha 12 (connexin 46.6) cause Pelizaeus-Merzbacher-like disease. Am J Hum Genet 2004; 75: 251-260.

4 Bugiani M, Al Shahwan S, Lamantea E et al: GJA12 mutations in children with recessive hypomyelinating leukoencephalopathy. Neurology 2006; 67 . 273-279.

5 Salviati L, Trevisson E, Baldoin MC: A novel deletion in the GJA12 gene causes Pelizaeus-Merzbacher-like disease. Neurogenet 2007; 8: 57-60.

6 Wolf NI, Cundall M, Rutland P et al: Frameshift mutation in GJA12 leading to nystagmus, spastic ataxia and CNS dys-/demyelination. Neurogenet 2007; 8: 39-44.

7 Henneke M, Combes P, Diekmann S et al: GJA12 mutations are a rare cause of Pelizaeus-Merzbacher-like disease. Neurology 2008; 70: 748-754.

8 Orthmann-Murphy JL, Salsano E, Abrams CK et al: Hereditary spastic paraplegia is a novel phenotype for GJA12/GJC2 mutations. Brain 2009; 132: 426-438.

9 Wang J, Wang H, Wang Y, Chen T, Wu X, Jiang Y: Two novel gap junction protein alpha 12 gene mutations in two Chinese patients with Pelizaeus-Merzbacher-like disease. Brain Dev 2009; 32: 236-243.

10 Trosko JE, Ruch RJ: Cell-cell communication in carcinogenesis. Front Biosci 1998; 3: D208-D236.

11 Kumar NM, Gilula NB: The gap junction communication channel. Cell 1996; 84: 381-388.

12 Nagy JI, Dudek FE, Rash JE: Update on connexins and gap junctions in neurons and glia in the mammalian nervous system. Brain Res Brain Res Rev 2004; 47: 191-215

13 Rash JE, Yasumura T, Dudek FE, Nagy JI: Cell-specific expression of connexins and evidence of restricted gap junctional coupling between glial cells and between neurons. J Neurosci 2001; 21: 1983-2000.

14 Massa PT, Mugnaini E: Cell junctions and intramembrane particles of astrocytes and oligodendrocytes: a freeze-fracture study. Neuroscience 1982; 7: 523-538.

15 Nagy JI, Ochalski PAY, Li J, Hertzberg EL: Evidence for the colocalization of another connexin with connexin-43 at astrocytic gap junctionsin rat brain. Neuroscience 1997; 78: 533-548.

16 Menichella DM, Goodenough DA, Sirkowski E, Scherer SS, Paul DL: Connexins are critical for normal myelination in the CNS. J Neurosci 2003; 23: 5963-5973.

17 Odermatt B, Wellershaus K, Wallraff A et al: Connexin $47(\mathrm{C} \times 47)$-deficient mice with enhanced green fluorescent protein reporter gene reveal predominant oligodendrocytic expression of cx47 and display vacuolized myelin in the cns. J Neurosci 2003; 23 : 4549-4559.

18 Giaume C, Fromaget C, El Aoumari A, Cordier J, Glowinski J, Gros D: Gap junctions in cultured astrocytes: single-channel currents and characterization of channel-forming protein. Neuron 1991; 6: 133-143.

19 Nagy JI, Li X, Rempel J et al: Connexin26 in adult rodent central nervous system: demonstration at astrocytic gap junctions and colocalization with connexin30 and connexin43. J Comp Neurol 2001; 441: 302-323.

20 Orthmann-Murphy JL, Freidin M, Fischer E, Scherer SS, Abrams CK: Two distinct heterotypic channels mediate gap junction coupling between astrocyte and oligodendrocyte connexins. J Neurosci 2007b; 27: 13949-13957.

21 Orthmann-Murphy JL, Enriquez AD, Abrams CK, Scherer SS: Loss-of-function GJA12/ Connexin47 mutations cause Pelizaeus-Merzbacher-like disease. Mol Cell Neurosci 2007a; 34: 629-641.
22 Jung $M$, Krämer E, Grzenkowski $M$ et al: Lines of murine oligodendroglial precursor cells immortalized by an activated neu tyrosine kinase show distinct degrees of interaction with axons in vitro and in vivo. Eur J Neurosci 1995; 7: 1245-1265.

23 Barrio LC, Suchyna T, Bargiello T et al: Gap junctions formed by connexins 26 and 32 alone and in combination are differently affected by applied voltage. Proc Natl Acad Sci USA 1991; 88: 8410-8414.

24 Barish ME: A transient calcium-dependent chloride current in the immature Xenopus oocyte. J Physiol 1983; 342: 309-325.

25 Ebihara L: New roles for connexons. News Physiol Sci 2003; 18: 100-103.

26 Kozak M: Interpreting cDNA sequences: some insights from studies. Mamm Genome 1996; 7: 563-574.

27 Foote $\mathrm{Cl}$, Zhou L, Zhu X, Nicholson BJ: The pattern of disulfide linkages in the extracellular loop regions of connexin 32 suggests a model for the docking interface of gap junctions. J Cell Biol 1998; 140: 1187-1197.

28 Dhaunchak AS, Nave KA: A common mechanism of PLP/DM20 misfolding causes cysteine-mediated endoplasmic reticulum retention in oligodendrocytes and Pelizaeus-Merzbacher disease. Proc Natl Acad Sci USA 2007; 104: $17813-17818$.

29 Olbina G, Eckhart W: Mutations in the second extracellular region of connexin43 prevent localization to the plasma membrane, but do not affect its ability to suppress cell growth. Mol Cancer Res 2003; 1: 690-700.

30 Moreno AP, Lau AF: Gap junction channel gating modulated through protein phosphorylation. Prog Biophys Mol Biol 2007; 94: 107-119.

31 Britz-Cunningham SH, Shah MM, Zuppan CW, Fletcher WH: Mutations of the connexin 43 gap-junction gene in patients with heart malformations and defects of laterality. New Engl J Med 1995; 332: 1323-1329.

32 Omori Y, Mesnil M, Yamasaki H: Connexin 32 mutations from X-linked Charcot-MarieTooth disease patients: functional defects and dominant negative effects. $\mathrm{Mol} \mathrm{BiO}$ Cell 1996; 7: 907-916.

33 Castro C, Gómez-Hernandez JM, Silander K, Barrio LC: Altered formation of hemichannels and gap junction channels caused by $\mathrm{C}$-terminal connexin32 mutations. J Neurosci 1999; 19: 3752-3760.

34 Kondo RP, Wang SY, John SA, Weiss JN, Goldhaber JI: Metabolic inhibition activates a non-selective current through connexin hemichannels in isolated ventricular myocytes. J Mol Cell Cardiol 2000; 32: 1859-1872.

35 Liang GS, de Miguel M, Gómez-Hernández JM et al: Severe neuropathy with leaky connexin32 hemichannels. Ann Neurol 2005; 57: 749-754.

36 Deschênes SM, Walcott JL, Wexler TL, Scherer SS, Fischbeck KH: Altered trafficking of mutant connexin32. J Neurosci 1997; 17: 9077-9084.

37 Orthmann-Murphy JL, Abrams CK, Scherer SS: Gap junctions couple astrocytes and oligodendrocytes. J Mol Neurosci 2008; 35: 101-116.

38 Gow A, Lazzarini RA: A cellular mechanism governing the severity of PelizaeusMerzbacher disease. Nat Genet 1996; 13: 422-428.

39 Gow A, Southwood CM, Lazzarini RA: Disrupted proteolipid protein trafficking results in oligodendrocyte apoptosis in an animal model of Pelizaeus-Merzbacher disease. J Cell Biol 1998; 140: 925-934.

40 D'Urso D, Prior R, Greiner-Petter R, Gabreëls-Festen AA, Müller HW: Overloaded endoplasmic reticulum-Golgi compartments, a possible pathomechanism of peripheral neuropathies caused by mutations of the peripheral myelin protein PMP22. J Neurosci 1998; 18: 731-740.

41 Aridor M, Balch WE: Integration of endoplasmic reticulum signaling in health and disease. Nat Med 1999; 5: 745-751.

42 Gow A, Friedrich VL, Lazzarini RA: Many naturally occurring mutations of myelin proteolipid protein impair its intracellular transport. J Neurosci Res 1994; 37: 574-583.

Supplementary Information accompanies the paper on European Journal of Human Genetics website (http://www.nature.com/ejhg) 